\title{
Natural Frequencies of Beams with Axial Material Gradation Resting on Two Parameter Elastic Foundation
}

\author{
Saurabh Kumar \\ Department of Mechanical Engineering, School of Engineering, University of Petroleum and Energy \\ Studies, Dehradun 248007, India
}

(Corresponding author's e-mail: saurabhks88@gmail.com)

Received: 8 April 2021, Revised: 13 June 2021, Accepted: 18 June 2021

\begin{abstract}
Free vibration analysis is carried out on axially inhomogeneous beams resting on Winkler-Pasternak elastic foundation. The material properties of the beam like Young's modulus, modulus of rigidity and material density are considered to be varying along the length direction following constant, linear and exponential material models. The beam is subjected to different combinations of clamped and simply supported boundary conditions. The formulation is based on Timoshenko beam theory and energy method along with Hamilton's principle is used to derive the governing equations. The effect of material gradation and the 2 parameters of elastic foundation on the natural frequencies are studied in detail. The present results are validated by comparing them with established ones and satisfactory matching is observed.
\end{abstract}

Keywords: Axially functionally graded beam, Timoshenko beam, Free vibration, Elastic foundation, Energy method

\section{Introduction}

Functionally graded materials (FGMs) are popular due to their distinct advantages over traditional composites. These advanced materials can be classified on the basis of the direction of material variation. FGMs with material variation in thickness/transverse direction have always been in focus since its inception however, in recent years a new type of FGMs has come into picture in which the material properties vary along the length direction called the axially functionally graded material (AFGM). These materials may find their application in structures where the optimisation of strength along the longitudinal direction is more important than transverse direction such as Helicopter rotor blades and aircraft antennae.

With such appealing prospects, the AFGMs naturally attracted the attention of researchers. Huang and $\mathrm{Li}$ [1] investigated the free vibration behaviour of non-uniform beams made of AFGM using Fredholm integral equations. This new approach was simple and gave fast convergence and accurate results. The same approach was also used to study their buckling behaviour [2]. Hein and Feklistova [3] investigated similar beams using Haar wavelets where the governing equations were transformed with the help of simple wavelets. Alshorbagy et al. [4] carried out dynamic analysis on beams with power law based axial material gradation. The beam was discretised using finite element method and Euler-Bernoulli beam theory in conjunction with principle of virtual work was used for deriving the equation of motion. Another novel approach was presented by Shahba and Rajasekaran [5] called differential transform element method which was derived from differential transform method to study the free vibration and stability of AFGM beams. Simsek et al. [6] derived the equation of motion of Euler-Bernoulli AFGM beams using Lagrange's equation and employed Newmark method to determine the forced vibration response of such beams. Nguyen [7] performed large displacement analysis of non-uniform AFGM beams and found out that the nonlinear behaviour is governed by material distribution, taper type and taper ratio. Li et al. [8] derived closed-form characteristics equations for exponentially graded beams and noted that the harmonic vibration can only be excited when bending waves with frequencies exceeding the critical value. Sarkar and Ganguli [9] provided closed form solutions of AFGM beams where the material was graded following certain polynomial functions. Bambill et al. [10] used differential quadrature method and domain decomposition technique to carry out free vibration analysis on AFGM beam with geometrical stepped changes. Wang and $\mathrm{Wu}$ [11] investigated the effect of thermal loads on 
the dynamic response of AFGM beams subjected to moving harmonic loads. The governing equations were derived using Lagrange method and Newmark- $\beta$ method was used to calculate the dynamic response. Nonlocal strain gradient theory was employed by Li et al. [12] to study the bending, buckling and vibration behaviour of AFGM beams where generalized differential quadrature method was employed for solving these problems. Ghayesh [13] performed nonlinear forced vibration analysis on non-uniform AFGM beams utilising $3^{\text {rd }}$ order shear deformation theory and Galerkin's method where Hamilton's principle was used to derive the governing equations. Xie et al. [14] studied the dynamic response of an AFGM beam subjected to moving transverse and longitudinal harmonic forces using Lagrange's equations and Newmark method. Zhang et al. [15] used Jacobi polynomial theory to carry out free vibration analysis on AFGM beams where the beam was modelled using Euler-Bernoulli, Timoshenko and nonlocal strain gradient beam theories.

An important aspect of FGMs is its interaction with elastic foundation. In fact, such interaction between transversely graded FGMs and elastic foundation has received quite a lot of attention. Ying et al. [16] presented exact solutions for beams resting on Winkler-Pasternak elastic foundation using 2dimensional theory of elasticity where they expanded the state variables into an infinite trigonometric series to reduce the governing partial differential equations to ordinary equations. Yan et al. [17] employed Galerkin's method and Hamilton's principle to investigate the dynamic response of FGM beam with an edge crack. The beam was modelled as 2 sub-beams joined together via linear rotational spring. Fallah and Aghdam [18] obtained approximate closed form solutions for nonlinear free vibration and post-buckling problem of FGM beams using He's variation method. They used the similar approach to investigate the thermo-mechanical buckling of such beams in their later work [19]. The same was also studied by Yaghoobi and Torabi [20,21] for normal and imperfect beams using Galerkin's method and variational iteration method. Kanani et al. [22] carried out free and forced vibration analysis of FGM beams considering geometric nonlinearity. Euler-Bernoulli beam theory and von Karman's nonlinear equations were utilised for the formulation and variational iteration method was used to derive approximate closed form solutions. Wattanasakulpong and Mao [23] employed Chebyshev collocation method to study the dynamic response of FGM beams where different models for material gradation were considered. Deng et al. [24] carried out vibration and buckling analysis on FGM double-beam system. The governing equations were derived using Hamilton's principle and Wittrick-William algorithm was used to determine the natural frequency and buckling load. Yas et al. [25] performed free vibration analysis on FGM beams resting on variable elastic foundation using Generalized Differential Quadrature method. Similar analysis was also performed by Avcar and Mohammed [26] using the method of separation of variables. Mohamed et al. [27] carried out nonlinear free and forced vibration analysis on buckled curved beams using differential-integral quadrature method. Nebab et al. [28] carried out static analysis on FGM plates subjected to mechanical loads using 4 variable shear deformation theory and principle of virtual work. Esen [29] presented a modified finite element method capable of analysing dynamic behaviour of FGM Timoshenko beams subjected to moving mass. Wattanasakulpong [30] performed free and forced vibration analysis on FG sandwich beams supported on Pasternak foundation using Ritz and Newmark method where Lagrange's equations were used to derive the equation of motion. Uzun and Yayli [31] used Eringen's nonlocal elasticity theory in conjunction with finite element method to investigate free vibration of FGM nanobeams in which material properties were varying according to the power law.

It is to be noted that, the works described above deal with transversely functionally graded material beams i.e., beams in which the material properties vary along the thickness direction. There are a couple of works on AFGM beams as well by Lohar et al. [32] and Jena et al. [33] but these works deal with 1 parameter elastic foundation, the effect of shearing layer was not considered. The present paper is motivated by the lack of research works on AFGM beams resting on 2 parameter elastic foundation and aims to bridge this research gap. As such, free vibration analysis is carried out on AFGM Timoshenko beams resting on Winkler-Pasternak elastic foundation and subjected to different end conditions. The methodology involves energy method with variational approach where Rayleigh-Ritz method is used to generate approximate displacement fields and Hamilton's principle is employed to derive the governing equations. The results are validated with those available in the literature and new results are presented in nondimensional form. The effect of material gradation, foundation parameters and boundary conditions on the free vibration behaviour of AFGM beams are discussed in detail. 


\section{Materials and methods}

An axially functionally graded beam of length $L$, cross-section A $(=b \times h)$ and $2^{\text {nd }}$ moment of area $I$ $\left(=b h^{3} / 12\right)$, and supported on a Winkler-Pasternak elastic foundation is shown in Figure 1. The material properties of the beam i.e., elastic modulus, modulus of rigidity and material density vary continuously along the length direction. Three different material models are considered namely; $F G M 1: E(x)=E_{0}, \rho(x)$

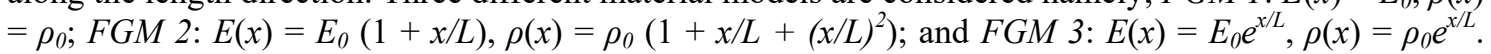
Here, $E_{0}$ and $\rho_{0}$ are the Young's modulus and material density respectively at one end of the beam. The modulus of rigidity is given by $G(\mathrm{x})=E(x) / 2(1+\mu)$ whereas the Poisson's ratio is assumed to remain constant. The beam is resting on a 2 parameter elastic foundation having Winker foundation modulus $K_{w}$ and shear foundation modulus $K_{p}$.

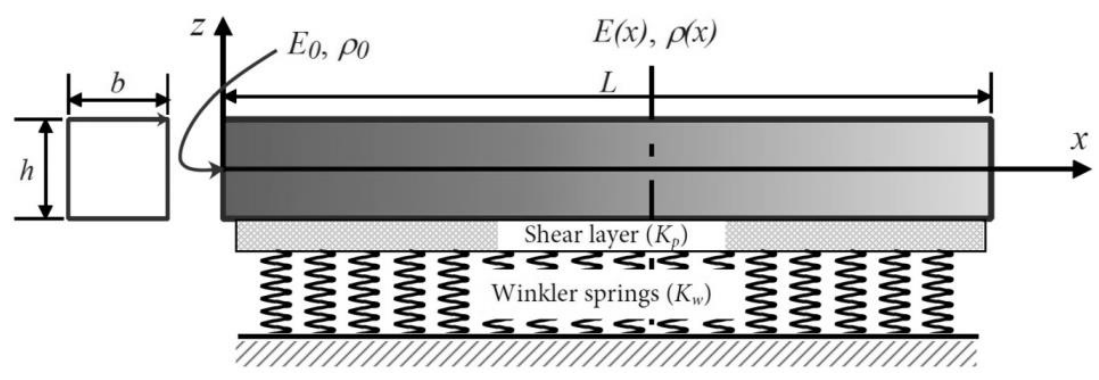

Figure 1 Axially functionally graded beam supported on 2 parameter elastic foundation.

A displacement based approximate approach is followed where appropriate displacement fields are assumed and substituted in the energy expressions to derive the governing equation of the dynamic system. The strain displacement relations of a Timoshenko beam where the effects of shear deformation and rotary inertia are accounted for are given as,

$\varepsilon_{x x}=\frac{d u}{d x}-z \frac{d \theta}{d x}, \gamma_{x z}=\frac{d v}{d x}-\theta$

where, $\varepsilon$ and $\gamma$ are the normal and shear strains, and $u, v$ and $\theta$ are axial, transverse and rotary displacements respectively. The axial $(\sigma)$ and shear stresses $(\tau)$ can be obtained from the elastic constitutive relationship as,

$\sigma_{x x}=E(x) \varepsilon_{x x}, \tau_{x z}=G(x) \gamma_{x z}$

The total strain energy of the beam resting on 2 parameter elastic foundation will be the summation of strain energy due to axial strain $\left(U_{\text {axial }}\right)$, strain energy due to shear strain $\left(U_{\text {shear }}\right)$ and the strain energy of the foundation $\left(U_{\text {foundation }}\right)$. Moreover, the strain energy of the foundation itself is the summation of the strain energy of the Winkler springs $\left(U_{w}\right)$ and the strain energy of the shearing layer $\left(U_{p}\right)$. So, the total strain energy of the system can be written as,

$U=U_{\text {axial }}+U_{\text {shear }}+U_{\text {foundation }}$

where, $U_{\text {foundation }}=U_{w}+U_{p}$.

These different strain energy components are given as,

$$
\begin{aligned}
& U_{\text {axial }}=\frac{1}{2} \int_{V} \sigma_{x x} \varepsilon_{x x} d V=\frac{1}{2} A \int_{0}^{L}\left(\frac{d u}{d x}\right)^{2} E(x) d x+\frac{1}{2} I \int_{0}^{L}\left(\frac{d \theta}{d x}\right)^{2} E(x) d x \\
& U_{\text {shear }}=\frac{1}{2} \int_{V} \tau_{x z} \gamma_{x z} d V=\frac{k_{s h}}{2} A \int_{0}^{L}\left[\left(\frac{d v}{d x}\right)^{2}+\theta^{2}-2 \theta\left(\frac{d v}{d x}\right)\right] G(x) d x
\end{aligned}
$$


$U_{w}=\frac{1}{2} \int_{0}^{L} K_{w} v^{2} d x$

$U_{p}=\frac{1}{2} \int_{0}^{L} K_{p}\left(\frac{d v}{d x}\right)^{2} d x$

where, $k_{s h}$ is the shear correction factor.

The expression of the kinetic energy of the present dynamic system is as follows,

$T=\frac{1}{2} A \int_{0}^{L}\left(\left(\frac{d u}{d t}\right)^{2}+\left(\frac{d v}{d t}\right)^{2}+z^{2}\left(\frac{d \theta}{d t}\right)^{2}\right) \rho(x) d x$

The computations are carried out in a normalised coordinate system given by $\xi=x / L$. A number of computational points are generated throughout the domain to serve as reference points for computation. Following the Rayleigh-Ritz approach the displacement fields are approximated as combinations of unknown parameters and orthogonally admissible functions. The properties of these functions are that they are continuous and differentiable within the domain and satisfy the respective boundary conditions. These orthogonal functions are represented by $\left(\alpha_{i}\right),\left(\beta_{i}\right)$ and $\left(\phi_{i}\right)$ for $u, v$ and $\theta$ respectively and given as,

$u(\xi, t)=\sum_{i=1}^{m u} d_{i} \alpha_{i}(\xi) e^{j \omega t}, v(\xi, t)=\sum_{i=n u+1}^{n u+n v} d_{i} \beta_{i}(\xi) e^{j \omega t}, \theta(\xi, t)=\sum_{i=1+n u+n v}^{n u+n v+n s} d_{i} \phi_{i}(\xi) e^{j \omega t}$

here, $\omega$ is the natural frequency of the system and $d_{i}$ is the unknown parameter. $n u, n v$ and $n s$ are the number of higher order functions for $\left(\alpha_{i}\right),\left(\beta_{i}\right)$ and $\left(\phi_{i}\right)$. Appropriate start functions are chosen for these higher order functions considering the properties mentioned above. The start function $\left(\alpha_{1}\right)$ for different boundary conditions is given as $\{\xi(1-\xi)\}^{2}$ for CC, $\xi^{2}\left(1.5-2.5 \xi+\xi^{2}\right)$ for CS and $\sin (\pi \xi)$ for SS; $\left(\beta_{l}\right)$ is given as $\xi(1-\xi)$ for all boundary conditions; $\left(\phi_{1}\right)$ is given as $\sin (\pi \xi)$ for $\mathrm{CC}, \sin (\pi \xi / 2)$ for CS and $\cos (\pi \xi)$ for SS.

After the energy expressions have been obtained the next step is to derive the governing equations of the dynamic problem. This is done using Hamilton's principle which states that,

$\delta\left(\int_{t_{1}}^{t_{2}}(T-U) d t\right)=0$

where, $\delta$ is the variational operator. Putting the energy expressions along with the dynamic displacement fields in the above equations gives,

$$
\begin{aligned}
& {\left[A L \int_{0}^{1} \alpha_{i} \alpha_{j} \rho(\xi) d \xi+A L \int_{0}^{1} \beta_{i} \beta_{j} \rho(\xi) d \xi+I L \int_{0}^{1} \phi_{i} \phi_{j} \rho(\xi) d \xi\right] \omega^{2}} \\
& -\left[\frac{A}{L} \int_{0}^{1} \frac{d \alpha_{i}}{d \xi} \frac{d \alpha_{j}}{d \xi} E(\xi) d \xi+\frac{I}{L} \int_{0}^{1} \frac{d \phi_{i}}{d \xi} \frac{d \phi_{j}}{d \xi} E(\xi) d \xi+\frac{k_{s h} A}{L} \int_{0}^{1} \frac{d \beta_{i}}{d \xi} \frac{d \beta_{j}}{d \xi} G(\xi) d \xi\right. \\
& -k_{s h} A \int_{0}^{1} \frac{d \beta_{i}}{d \xi} \phi_{j} G(\xi) d \xi-k_{s h} A \int_{0}^{1} \phi_{i} \frac{d \beta_{j}}{d \xi} G(\xi) d \xi+k_{s h} A L \int_{0}^{1} \phi_{i} \phi_{j} G(\xi) d \xi \\
& \left.+K_{w} L \int_{0}^{1} \beta_{i} \beta_{j} d \xi+\frac{K_{p}}{L} \int_{0}^{1} \frac{d \beta_{i}}{d \xi} \frac{d \beta_{j}}{d \xi} d \xi\right]=0
\end{aligned}
$$

The above expression can be written in following form,

$$
[K]-\omega^{2}[M]=0
$$


where, $[K]$ is the stiffness matrix and $[M]$ is the mass matrix of the system. These matrices are calculated from the following expressions,

$$
\begin{aligned}
{[K]=} & {\left[\frac{A}{L} \int_{0}^{1} \frac{d \alpha_{i}}{d \xi} \frac{d \alpha_{j}}{d \xi} E(\xi) d \xi+\frac{I}{L} \int_{0}^{1} \frac{d \phi_{i}}{d \xi} \frac{d \phi_{j}}{d \xi} E(\xi) d \xi+\frac{k_{s h} A}{L} \int_{0}^{1} \frac{d \beta_{i}}{d \xi} \frac{d \beta_{j}}{d \xi} G(\xi) d \xi\right.} \\
& -k_{s h} A \int_{0}^{1} \frac{d \beta_{i}}{d \xi} \phi_{j} G(\xi) d \xi-k_{s h} A \int_{0}^{1} \phi_{i} \frac{d \beta_{j}}{d \xi} G(\xi) d \xi+k_{s h} A L \int_{0}^{1} \phi_{i} \phi_{j} G(\xi) d \xi \\
& \left.+K_{w} L \int_{0}^{1} \beta_{i} \beta_{j} d \xi+\frac{K_{p}}{L} \int_{0}^{1} \frac{d \beta_{i}}{d \xi} \frac{d \beta_{j}}{d \xi} d \xi\right] \\
{[M]=} & {\left[A L \int_{0}^{1} \alpha_{i} \alpha_{j} \rho(\xi) d \xi+A L \int_{0}^{1} \beta_{i} \beta_{j} \rho(\xi) d \xi+I L \int_{0}^{1} \phi_{i} \phi_{j} \rho(\xi) d \xi\right] }
\end{aligned}
$$

Eq. (12) is a standard eigenvalue problem and its solution gives the natural frequency of the system.

\section{Results and discussion}

Dynamic analysis is conducted on AFG Timoshenko beams resting on 2 parameter elastic foundation. Three different functionally graded material models are investigated considering different combinations of clamped (C) and simply supported (S) boundary conditions for different values of foundation parameters and slenderness ratio. Based on the convergence study performed by Kumar et al. [34] the number of higher order functions is taken as 8 for the approximation of the displacement fields and the number of computational points throughout the domain is taken as 24 . The results are presented in nondimensional form and the various nondimensional parameters are calculated as,

$$
s_{r}=\frac{L}{h}, \bar{\omega}=\omega L^{2} \sqrt{\frac{\rho_{0} A}{E_{0} I}}, \bar{K}_{w}=\frac{K_{w} L^{4}}{E_{0} I}, \text { and } \bar{K}_{p}=\frac{K_{p} L^{2}}{E_{0} I}
$$

The methodology presented in this paper is validated with the results published by Chen et al. [35] and furnished in Figure 2. These results are generated for homogeneous (FGM 1) beams with clampedclamped (CC) and simply supported-simply supported (SS) boundary conditions. Two values of slenderness ratio $\left(s_{r}\right), 120$ and 5 , associated with thin beam and thick beam respectively and different combinations of foundation stiffness parameters $\left(\bar{K}_{w}\right.$ and $\left.\bar{K}_{P}\right)$ are considered. It can be seen that, the present results match reasonably with established results.
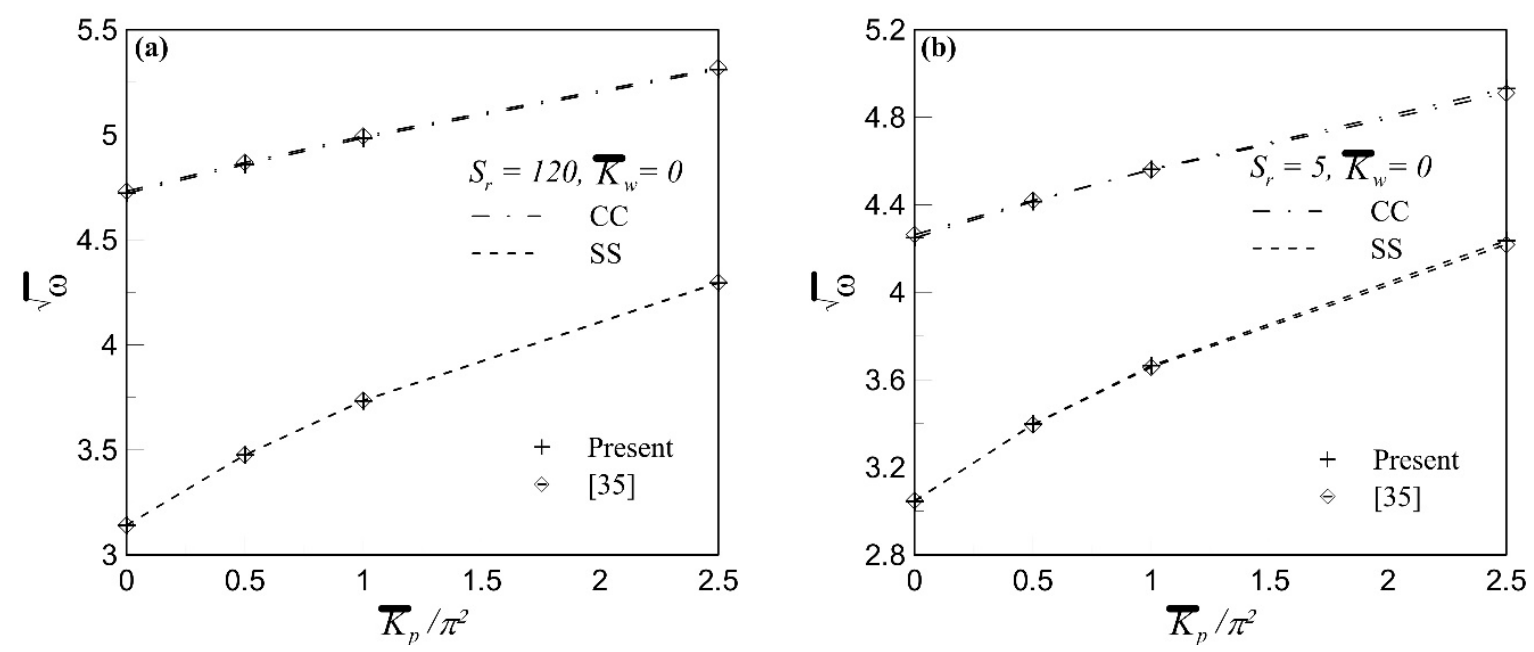

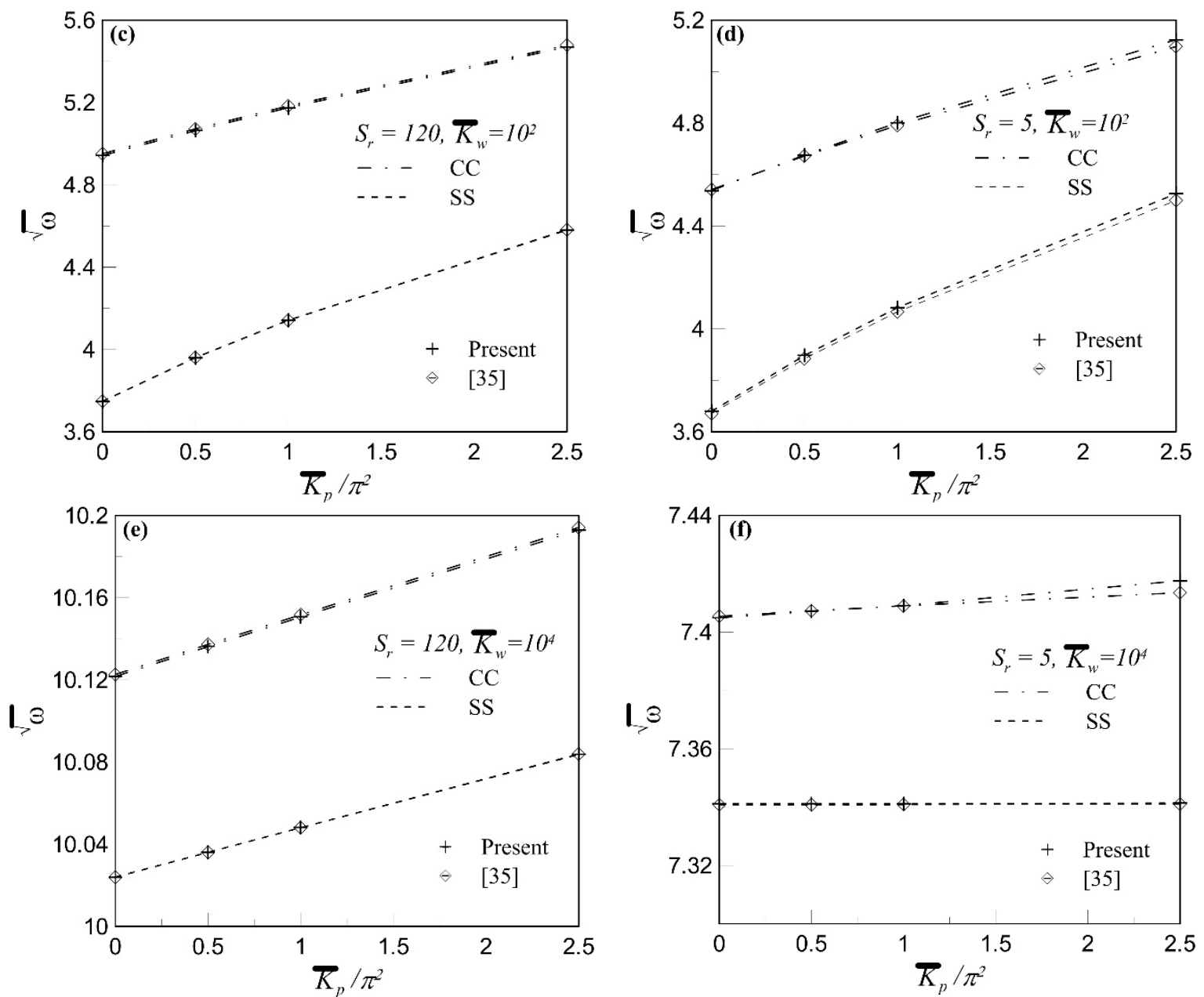

Figure 2 Comparison of non-dimensional frequency parameters of homogeneous material (FGM 1) beam.

Nondimensional frequency parameters of linearly AFG (FGM 2) beam and exponentially AFG beam $(F G M 3)$ are furnished in Tables 1 and $\mathbf{2}$ respectively. These results are generated for beams with slenderness ratios 100, 50 and 10 and subjected to CC, CS and SS boundary conditions. Four values of $\bar{K}_{w}$ which are $0,10^{2}, 10^{3}$ and $10^{4}$ and 4 values of $\bar{K}_{P}$ which are $0,5,10$ and 25 are considered and the results are presented for different combinations of these values. In general, it is noticed that, increasing any of the foundation stiffness parameters increases the natural frequency of the system for all the cases i.e., for all material models, boundary conditions and slenderness ratios. This can be attributed to the overall increase in the stiffness of the system as a result of raising the foundation stiffness. An interesting behaviour is noticed when the frequency parameters for different slenderness ratios are compared. It is observed that, when $L / h$ ratio is decreased the frequency parameter also decreases which suggests that the added material does not increase the stiffness of the material as much as it increases its mass. Another observation is that, for similar conditions the frequency for CC boundary condition is highest and SS boundary condition is lowest which may be attributed to the fact that clamped ends provide additional stiffness to the structure. 
Table 1 Nondimensional frequency parameters of linearly AFG material (FGM 2) beam for different foundation parameters and $L / h$ ratios.

\begin{tabular}{|c|c|c|c|c|c|c|c|c|c|c|}
\hline \multirow{3}{*}{$\bar{K}_{w}$} & \multirow{3}{*}{$\bar{K}_{p}$} & \multicolumn{9}{|c|}{$\sqrt{\bar{\omega}}$} \\
\hline & & \multicolumn{3}{|c|}{$s_{r}=100$} & \multicolumn{3}{|c|}{$s_{r}=50$} & \multicolumn{3}{|c|}{$s_{r}=10$} \\
\hline & & $\mathrm{CC}$ & CS & SS & $\mathrm{CC}$ & $\mathrm{CS}$ & SS & $\mathrm{CC}$ & CS & SS \\
\hline \multirow{4}{*}{0} & 0 & 4.5154 & 3.6363 & 3.0073 & 4.5108 & 3.6342 & 3.0065 & 4.3773 & 3.5691 & 2.9821 \\
\hline & 5 & 4.6072 & 3.7805 & 3.2342 & 4.6028 & 3.7785 & 3.2335 & 4.4738 & 3.7180 & 3.2123 \\
\hline & 10 & 4.6935 & 3.9097 & 3.4213 & 4.6892 & 3.9078 & 3.4207 & 4.5639 & 3.8506 & 3.4013 \\
\hline & 25 & 4.9252 & 4.2343 & 3.8503 & 4.9211 & 4.2326 & 3.8497 & 4.8033 & 4.1813 & 3.8330 \\
\hline \multirow{4}{*}{$10^{2}$} & 0 & 4.6632 & 3.8838 & 3.4272 & 4.6590 & 3.8820 & 3.4266 & 4.5373 & 3.8278 & 3.4073 \\
\hline & 5 & 4.7468 & 4.0039 & 3.5871 & 4.7427 & 4.0022 & 3.5865 & 4.6242 & 3.9505 & 3.5684 \\
\hline & 10 & 4.8258 & 4.1139 & 3.7280 & 4.8218 & 4.1122 & 3.7274 & 4.7059 & 4.0624 & 3.7101 \\
\hline & 25 & 5.0402 & 4.3986 & 4.0753 & 5.0363 & 4.3970 & 4.0748 & 4.9258 & 4.3507 & 4.0588 \\
\hline \multirow{4}{*}{$10^{3}$} & 0 & 5.6012 & 5.1387 & 5.0324 & 5.5986 & 5.1377 & 5.0319 & 5.5225 & 5.1078 & 5.0153 \\
\hline & 5 & 5.6496 & 5.1931 & 5.0854 & 5.6469 & 5.1921 & 5.0848 & 5.5708 & 5.1624 & 5.0682 \\
\hline & 10 & 5.6966 & 5.2457 & 5.1367 & 5.6939 & 5.2447 & 5.1362 & 5.6176 & 5.2151 & 5.1194 \\
\hline & 25 & 5.8298 & 5.3939 & 5.2821 & 5.8271 & 5.3929 & 5.2816 & 5.7499 & 5.3631 & 5.2646 \\
\hline \multirow{4}{*}{$10^{4}$} & 0 & 8.7420 & 8.4438 & 8.5993 & 8.7396 & 8.4422 & 8.5965 & 8.6725 & 8.3940 & 8.5302 \\
\hline & 5 & 8.7559 & 8.4612 & 8.6115 & 8.7536 & 8.4596 & 8.6087 & 8.6871 & 8.4127 & 8.5438 \\
\hline & 10 & 8.7696 & 8.4781 & 8.6235 & 8.7673 & 8.4766 & 8.6208 & 8.7015 & 8.4309 & 8.5571 \\
\hline & 25 & 8.8099 & 8.5270 & 8.6591 & 8.8076 & 8.5256 & 8.6565 & 8.7431 & 8.4831 & 8.5958 \\
\hline
\end{tabular}

Table 2 Nondimensional frequency parameters of exponentially AFG material (FGM 3) beam for different foundation parameters and $L / h$ ratios.

\begin{tabular}{|c|c|c|c|c|c|c|c|c|c|c|}
\hline \multirow{3}{*}{$\bar{K}_{w}$} & \multirow{3}{*}{$\bar{K}_{p}$} & \multicolumn{9}{|c|}{$\sqrt{\bar{\omega}}$} \\
\hline & & \multicolumn{3}{|c|}{$s_{r}=100$} & \multicolumn{3}{|c|}{$s_{r}=50$} & \multicolumn{3}{|c|}{$s_{r}=10$} \\
\hline & & $\mathbf{C C}$ & CS & SS & $\mathrm{CC}$ & CS & SS & $\mathrm{CC}$ & CS & SS \\
\hline \multirow{4}{*}{0} & 0 & 4.7051 & 3.7878 & 3.1369 & 4.7303 & 3.7856 & 3.1360 & 4.5898 & 3.7161 & 3.1112 \\
\hline & 5 & 4.8216 & 3.9246 & 3.3521 & 4.8170 & 3.9224 & 3.3513 & 4.6810 & 3.8575 & 3.3295 \\
\hline & 10 & 4.9034 & 4.0481 & 3.5323 & 4.8988 & 4.0461 & 3.5316 & 4.7666 & 3.9845 & 3.5117 \\
\hline & 25 & 5.1249 & 4.3625 & 3.9517 & 5.1206 & 4.3606 & 3.9511 & 4.9964 & 4.3052 & 3.9340 \\
\hline \multirow{4}{*}{$10^{2}$} & 0 & 4.8732 & 4.0259 & 3.5379 & 4.8688 & 4.0240 & 3.5372 & 4.7397 & 3.9655 & 3.5173 \\
\hline & 5 & 4.9528 & 4.1412 & 3.6934 & 4.9484 & 4.1393 & 3.6928 & 4.8227 & 4.0834 & 3.6741 \\
\hline & 10 & 5.0283 & 4.2474 & 3.8313 & 5.0240 & 4.2456 & 3.8307 & 4.9011 & 4.1916 & 3.8130 \\
\hline & 25 & 5.2347 & 4.5248 & 4.1742 & 5.2307 & 4.5231 & 4.1337 & 5.1136 & 4.4729 & 4.1574 \\
\hline \multirow{4}{*}{$10^{3}$} & 0 & 5.7764 & 5.2691 & 5.1319 & 5.7735 & 5.2681 & 5.1314 & 5.6917 & 5.2365 & 5.1154 \\
\hline & 5 & 5.8242 & 5.3226 & 5.1852 & 5.8213 & 5.3216 & 5.1847 & 5.7396 & 5.2900 & 5.1687 \\
\hline & 10 & 5.8707 & 5.3744 & 5.2369 & 5.8678 & 5.3734 & 5.2363 & 5.7861 & 5.3418 & 5.2203 \\
\hline & 25 & 6.0028 & 5.5208 & 5.3833 & 5.9999 & 5.5197 & 5.3828 & 5.9178 & 5.4879 & 5.3665 \\
\hline \multirow{4}{*}{$10^{4}$} & 0 & 8.9457 & 8.6454 & 8.7683 & 8.9436 & 8.6440 & 8.7661 & 8.8840 & 8.6008 & 8.7136 \\
\hline & 5 & 8.9591 & 8.6614 & 8.7798 & 8.9570 & 8.6600 & 8.7777 & 8.8977 & 8.6179 & 8.7259 \\
\hline & 10 & 8.9723 & 8.6771 & 8.7913 & 8.9702 & 8.6757 & 8.7892 & 8.9112 & 8.6346 & 8.7381 \\
\hline & 25 & 9.0112 & 8.7226 & 8.8253 & 9.0091 & 8.7214 & 8.8233 & 8.9508 & 8.6827 & 8.7739 \\
\hline
\end{tabular}

The relationship between foundation parameter $\bar{K}_{w}$ and the nondimensional frequency parameter is shown in Figures 3 - 5 for CC, CS and SS boundary conditions respectively. These graphs are generated by gradually increasing the foundation parameter $\bar{K}_{w}$ from 0 to $10^{4}$ and calculating the frequency parameters at each increment. Different material models and boundary conditions are considered and the values of foundation parameter $\bar{K}_{P}$ are selected as $0,5,10$ and 25. A nonlinear relationship is observed 
where the slope of the curve gradually decreases as the value of $\bar{K}_{w}$ increases. The increase in natural frequency when $\bar{K}_{w}$ is increased from 0 to 2000 is around 40 to $50 \%$ for CC, 70 to $80 \%$ for CS and more than $100 \%$ for SS whereas when it is increased from 2000 to 4000 the increase in frequency is only 15 to $25 \%$ for all boundary conditions, it is even less when $\bar{K}_{w}$ is increased further. This suggests that the foundation parameter $\bar{K}_{w}$ does not have as much effect on the frequency parameter when its vales in on the higher side.
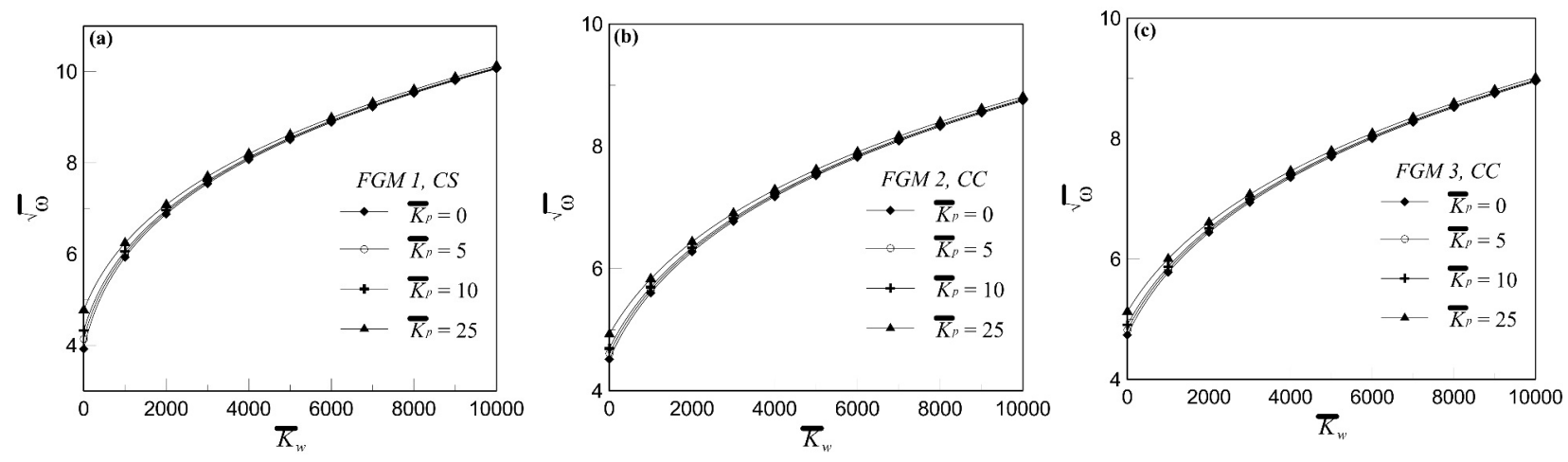

Figure 3 Relationship between foundation parameter $\bar{K}_{w}$ and nondimensional frequency parameter for CC boundary condition.
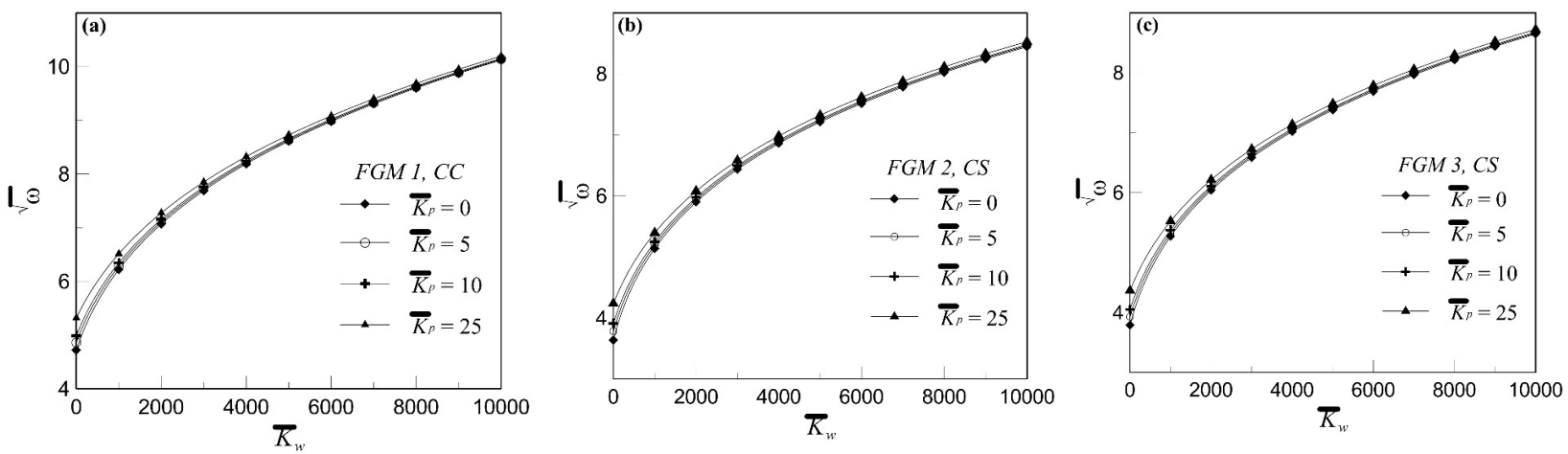

Figure 4 Relationship between foundation parameter $\bar{K}_{w}$ and nondimensional frequency parameter for CS boundary condition.
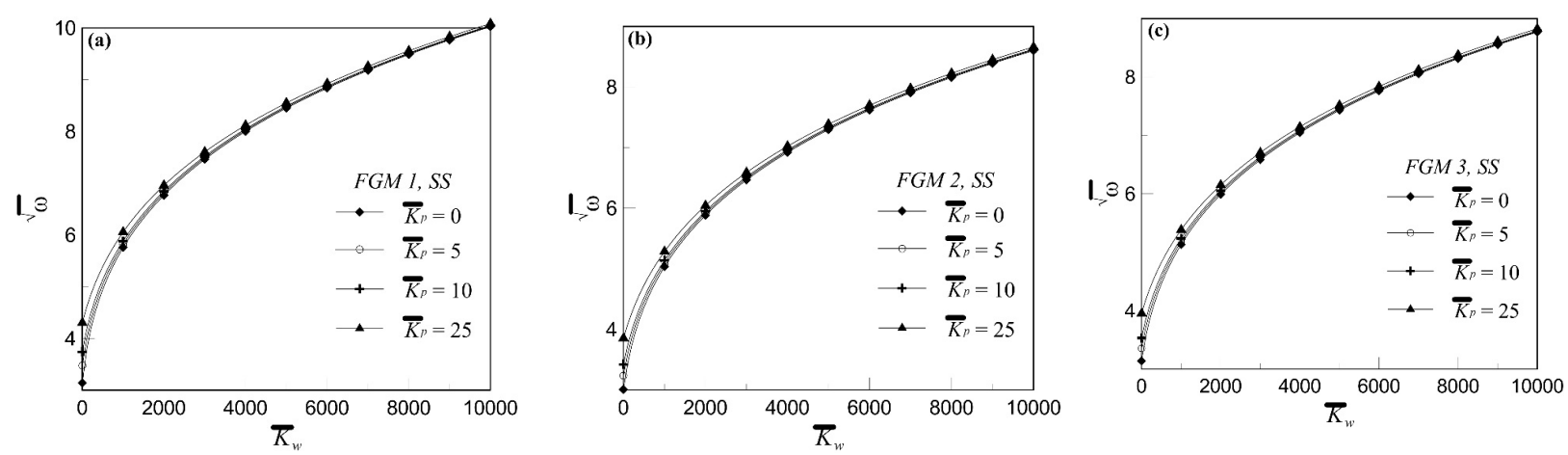

Figure 5 Relationship between foundation parameter $\bar{K}_{w}$ and nondimensional frequency parameter for SS boundary condition. 
Figures 6 - 8 show the relationship between foundation parameter $\bar{K}_{P}$ and nondimensional frequency parameter of AFG beam for CC, CS and SS boundary conditions respectively. The value of $\bar{K}_{P}$ is varied from 0 to 25 whereas 4 values of $\bar{K}_{W}\left(0,10^{2}, 10^{3}\right.$ and $\left.10^{4}\right)$ are considered for plotting the graphs. The relationship is shown for all 3 materials models (FGM 1, FGM 2 and $F G M 3$ ) and boundary conditions (CC, CS and SS). The natural frequency increases linearly when $\bar{K}_{P}$ is varied from 0 to 25 but the graph flattens for higher values of $\bar{K}_{W}$. When $\bar{K}_{W}=0$, the difference in natural frequency for $\bar{K}_{P}=0$ and $\bar{K}_{P}=25$ is around 12,20 and $35 \%$ for CC, CS and SS boundary conditions respectively, whereas this remains only 0.5 to $0.7 \%$ when $\bar{K}_{W}$ is $10^{4}$ for all boundary conditions. This suggests that the effect of $\bar{K}_{P}$ diminishes for higher values of $\bar{K}_{W}$, irrespective of the material models and boundary conditions.
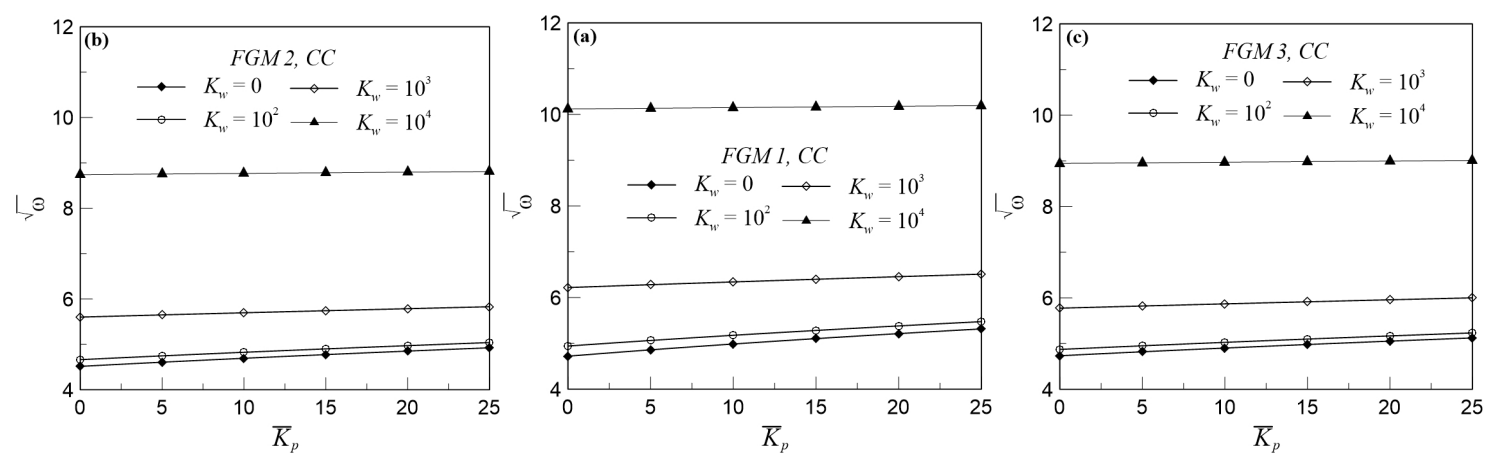

Figure 6 Relationship between foundation parameter $\bar{K}_{P}$ and nondimensional frequency parameter for CC boundary condition.
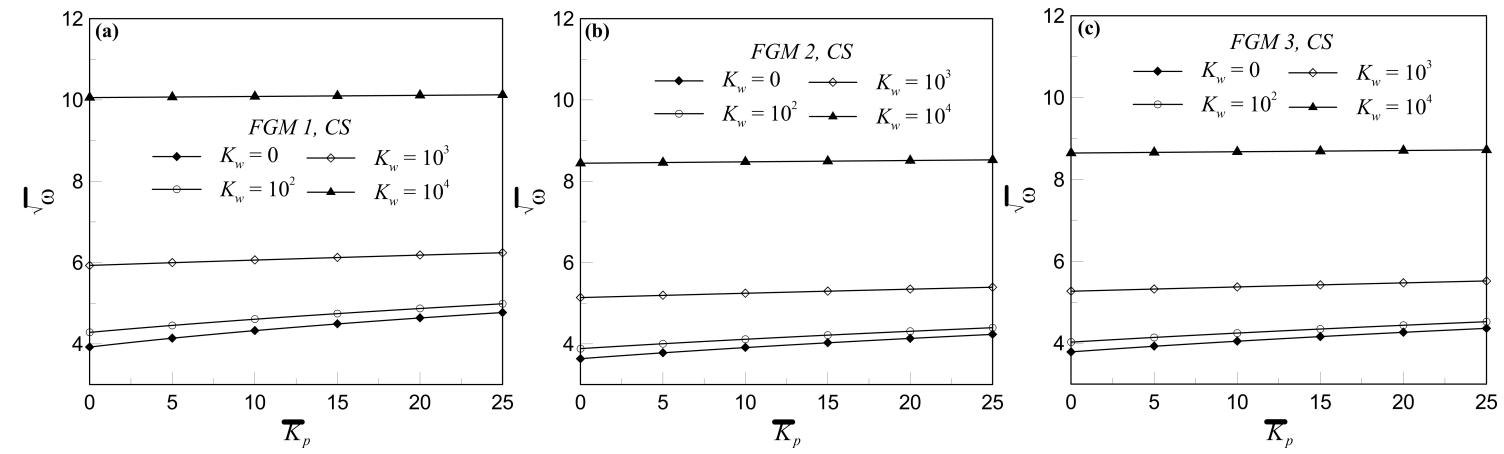

Figure 7 Relationship between foundation parameter $\bar{K}_{P}$ and nondimensional frequency parameter for CS boundary condition.
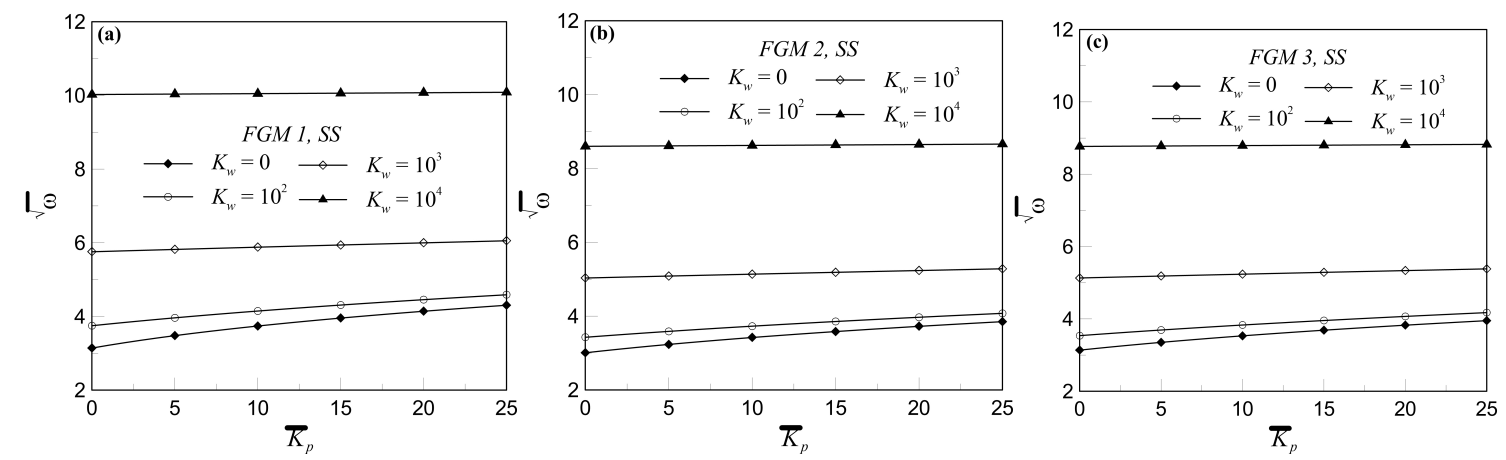

Figure 8 Relationship between foundation parameter $\bar{K}_{P}$ and nondimensional frequency parameter for SS boundary condition. 
The frequency parameters of different material models are compared in Figure 9 for different boundary conditions. Since it has been established that the effect of foundation parameter $\bar{K}_{W}$ is more significant than $\bar{K}_{P}$, the graphs are presented with respect to $\bar{K}_{W}$ and the value of $\bar{K}_{P}$ is kept constant. It is observed that, for similar conditions the frequency of $F G M 2$ is always less than that of other material models. For lower values of $\bar{K}_{W}$ the frequency values of all the material models are close to each other. However, as the value of $\bar{K}_{W}$ increases the graph of FGM 1 significantly diverges from the other models whereas the graphs of FGM 2 and FGM 3 remain close to each other. Similar analysis is shown in Figure 10, where the nondimensional frequency parameters of different boundary conditions are compared. It can be seen that, the frequency parameter for CC boundary condition is highest followed by CS boundary condition and it is lowest for SS boundary condition. However, this difference becomes insignificant for higher values of $\bar{K}_{W}$ which can be interpreted from the converging graphs as the value of $\bar{K}_{W}$ increases.
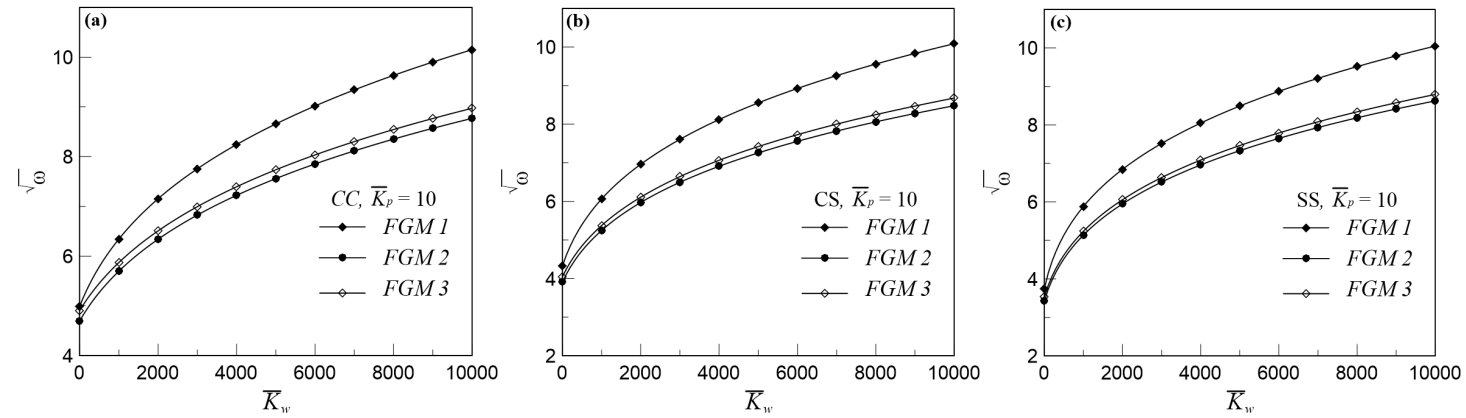

Figure 9 Comparison of nondimensional frequency parameters of different material models.
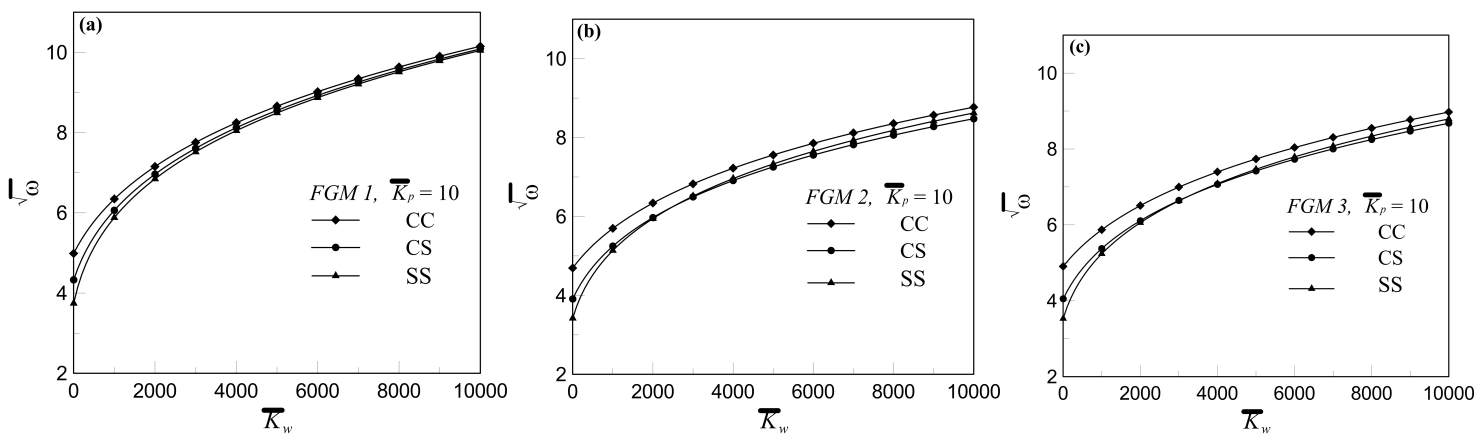

Figure 10 Comparison of nondimensional frequency parameters for different boundary conditions.

\section{Conclusions}

Free vibration analysis was conducted on axially functionally graded material beams resting on 2 parameter elastic foundation and subjected to different end conditions using energy method and variational approach. The approximate displacement fields were assumed using Rayleigh-Ritz method and Hamilton's principle was employed to derive the governing equations. The effect of material models, foundation parameters and boundary conditions on the dynamic behaviour of the beam is discussed in detail. It was found that, at the higher values of Winkler foundation parameter the effect of other parameters reduce significantly. The methodology was validated by comparing the present results with those already available in literature. The method is simple, fast and accurate, and can be modified to incorporate various complex scenarios. 


\section{References}

[1] Y Huang and XF Li. A new approach for free vibration of axially functionally graded beams with non-uniform cross-section. J. Sound Vib. 2010; 329, 2291-303.

[2] Y Huang and XF Li. Buckling analysis of nonuniform and axially graded columns with varying flexural rigidity. J. Eng. Mech. 2011; 137, 73-81.

[3] H Hein and L Feklistova. Free vibrations of non-uniform and axially functionally graded beams using Haar wavelets. Eng. Struct. 2011; 33, 3696-701.

[4] AE Alshorbagy, MA Eltaher and F Mahmoud. Free vibration characteristics of a functionally graded beam by finite element method. Appl. Math. Modell. 2011; 35, 412-25.

[5] A Shahba and S Rajasekaran. Free vibration and stability of tapered Euler-Bernoulli beams made of axially functionally graded materials. Appl. Math. Modell. 2012; 36, 3094-111.

[6] M Simsek, T Kocaturk and SD Akbas. Dynamic behavior of an axially functionally graded beam under action of a moving harmonic load. Compos. Struct. 2012; 94, 2358-64.

[7] DK Nguyen. Large displacement response of tapered cantilever beams made of axially functionally graded material. Compos. Part B: Eng. 2013; 55, 298-305.

[8] XF Li, YA Kang and JX Wu. Exact frequency equations of free vibration of exponentially functionally graded beams. Appl. Acoust. 2013; 74, 413-20.

[9] K Sarkar and R Ganguli. Closed-form solutions for axially functionally graded Timoshenko beams having uniform cross-section and fixed-fixed boundary condition. Compos. Part B: Eng. 2014; 58, 361-70.

[10] DV Bambill, CA Rossit and DH Felix. Free vibrations of stepped axially functionally graded Timoshenko beams. Meccanica 2015; 50, 1073-87.

[11] Y Wang and D Wu. Thermal effect on the dynamic response of axially functionally graded beam subjected to a moving harmonic load. Acta Astronaut. 2016; 127, 171-81.

[12] X Li, L Li, Y Hu, Z Ding and W Deng. Bending, buckling and vibration of axially functionally graded beams based on nonlocal strain gradient theory. Compos. Struct. 2017; 165, 250-65.

[13] MH Ghayesh. Nonlinear vibration analysis of axially functionally graded shear-deformable tapered beams. Appl. Math. Modell. 2018; 59, 583-96.

[14] K Xie, Y Wang and T Fu. Dynamic response of axially functionally graded beam with longitudinaltransverse coupling effect. Aerosp. Sci. Technol. 2019; 85, 85-95.

[15] X Zhang, Z Ye and Y Zhou. A Jacobi polynomial based approximation for free vibration analysis of axially functionally graded material beams. Compos. Struct. 2019; 225, 111070.

[16] J Ying, CF Lu and WQ Chen. Two-dimensional elasticity solutions for functionally graded beams resting on elastic foundations. Compos. Struct. 2008; 84, 209-19.

[17] T Yan, S Kitipornchai, J Yang and XQ He. Dynamic behaviour of edge-cracked shear deformable functionally graded beams on an elastic foundation under a moving load. Compos. Struct. 2011; 93, 2992-3001.

[18] A Fallah and MM Aghdam. Nonlinear free vibration and post-buckling analysis of functionally graded beams on nonlinear elastic foundation. Eur. J. Mech. A Solids 2011; 30, 571-83.

[19] A Fallah and MM Aghdam. Thermo-mechanical buckling and nonlinear free vibration analysis of functionally graded beams on nonlinear elastic foundation. Compos. Part B: Eng. 2012; 43, 152330 .

[20] H Yaghoobi and M Torabi. An analytical approach to large amplitude vibration and post-buckling of functionally graded beams rest on non-linear elastic foundation. J. Theor. Appl. Mech. 2013; 51, 39-52.

[21] H Yaghoobi and M Torabi. Post-buckling and nonlinear free vibration analysis of geometrically imperfect functionally graded beams resting on nonlinear elastic foundation. Appl. Math. Modell. 2013; 37, 8324-40.

[22] AS Kanani, H Niknam, AR Ohadi and MM Aghdam. Effect of nonlinear elastic foundation on large amplitude free and forced vibration of functionally graded beam. Compos. Struct. 2014; 115, 60-8.

[23] N Wattanasakulpong and Q Mao. Dynamic response of Timoshenko functionally graded beams with classical and non-classical boundary conditions using Chebyshev collocation method. Compos. Struct. 2015; 119, 346-54.

[24] H Deng, K Chen, W Cheng and S Zhao. Vibration and buckling analysis of double-functionally graded Timoshenko beam system on Winkler-Pasternak elastic foundation. Compos. Struct. 2017; 160, 152-68. 
[25] MH Yas, S Kamarian and A Pourasghar. Free vibration analysis of functionally graded beams resting on variable elastic foundations using a generalized power-law distribution and GDQ method. Ann. Solid Struct. Mech. 2017; 9, 1-11.

[26] M Avcar and WKM Mohammed. Free vibration of functionally graded beams resting on WinklerPasternak foundation. Arab. J. Geosci. 2018; 11, 232.

[27] N Mohamed, MA Eltaher, SA Mohamed and LF Seddek. Numerical analysis of nonlinear free and forced vibrations of buckled curved beams resting on nonlinear elastic foundations. Int. J. Non Linear Mech. 2018; 101, 157-73.

[28] M Nebab, HA Atmane, R Bennai and A Tounsi. Effect of variable elastic foundations on static behavior of functionally graded plates using sinusoidal shear deformation. Arab. J. Geosci. 2019; 12, 809 .

[29] I Esen. Dynamic response of a functionally graded Timoshenko beam on two-parameter elastic foundations due to a variable velocity moving mass. Int. J. Mech. Sci. 2019; 153-154, 21-35.

[30] N Wattanasakulpong. Multi-moving loads induced vibration of FG sandwich beams resting on Pasternak elastic foundation. Walailak J. Sci. Technol. 2021; 18, 9260.

[31] B Uzun and MO Yayli. Nonlocal vibration analysis of Ti-6Al-4V/Z $\mathrm{ZrO}_{2}$ functionally graded nanobeam on elastic matrix. Arab. J. Geosci. 2020; 13, 155.

[32] H Lohar, A Mitra and S Sahoo. Nonlinear response of axially functionally graded Timoshenko beams on elastic foundation under harmonic excitation. Curved Layer Struct. 2019; 6, 90-104.

[33] SK Jena, S Chakraverty and F Tornabene. Vibration characteristics of nanobeam with exponentially varying flexural rigidity resting on linearly varying elastic foundation using differential quadrature method. Mater. Res. Express 2019; 6, 085051.

[34] S Kumar, A Mitra and H Roy. Geometrically nonlinear free vibration analysis of axially functionally graded taper beams. Eng. Sci. Technol. 2015; 18, 579-93.

[35] WQ Chen, CF Lu and ZG Bian. A mixed method for bending and free vibration of beams resting on a Pasternak elastic foundation. Appl. Math. Modell. 2004; 28, 877-90. 